\title{
Supplementary Material
}

\section{Figures}

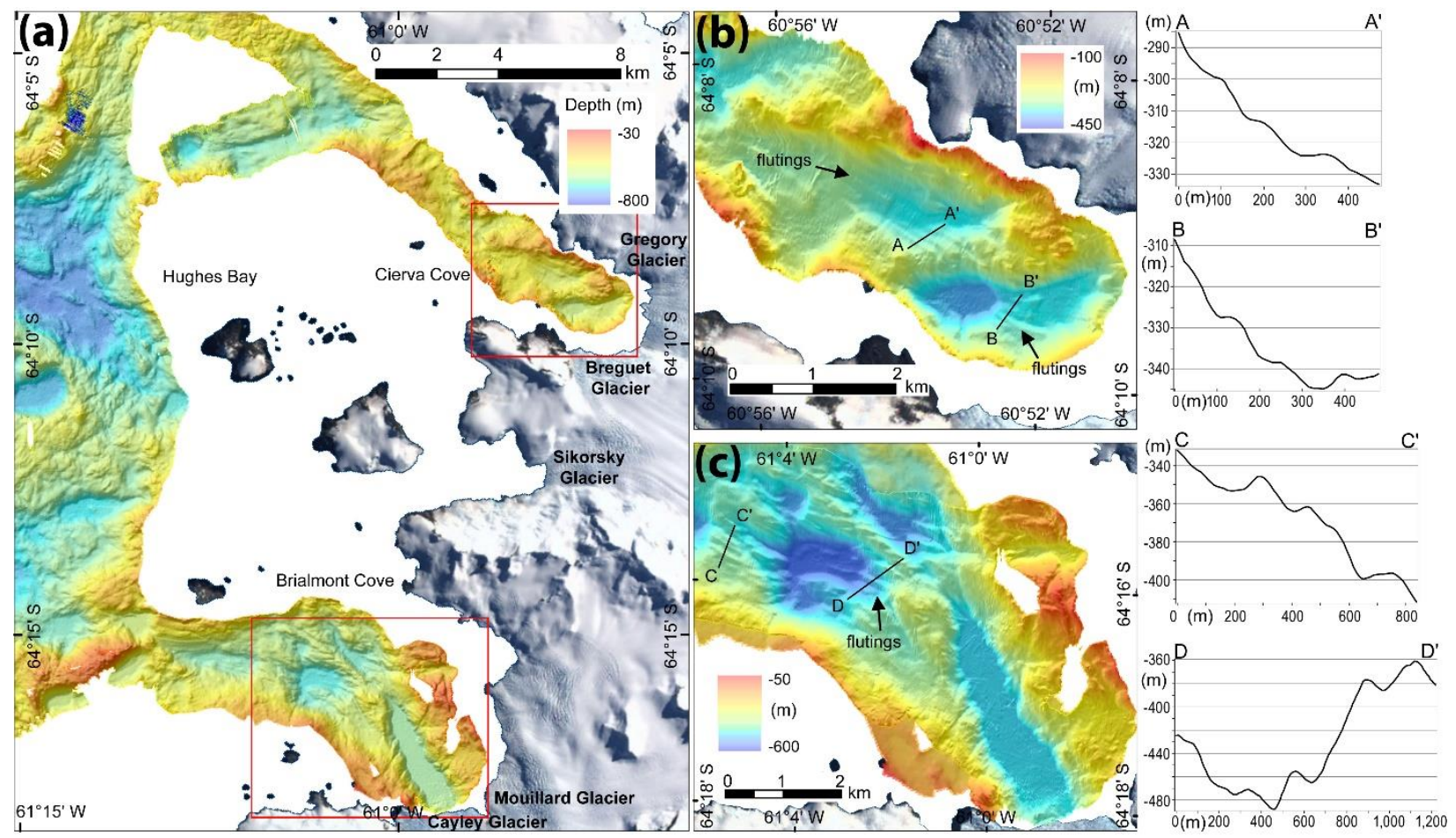

Figure 1: Hughes Bay, showing Cierva Cove (b) and Brialmont Cove (c). 


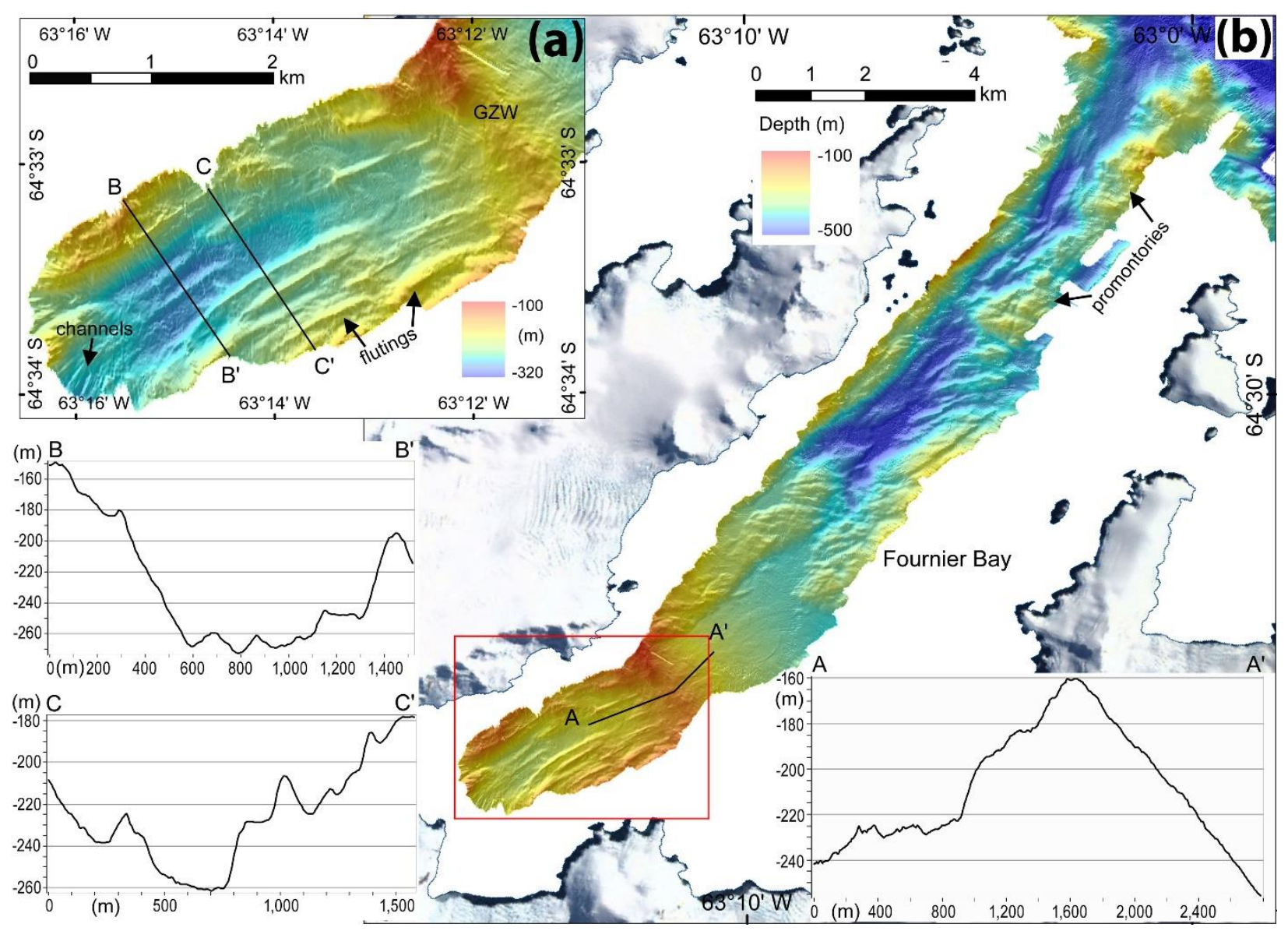

Figure 2: Fournier Bay. 


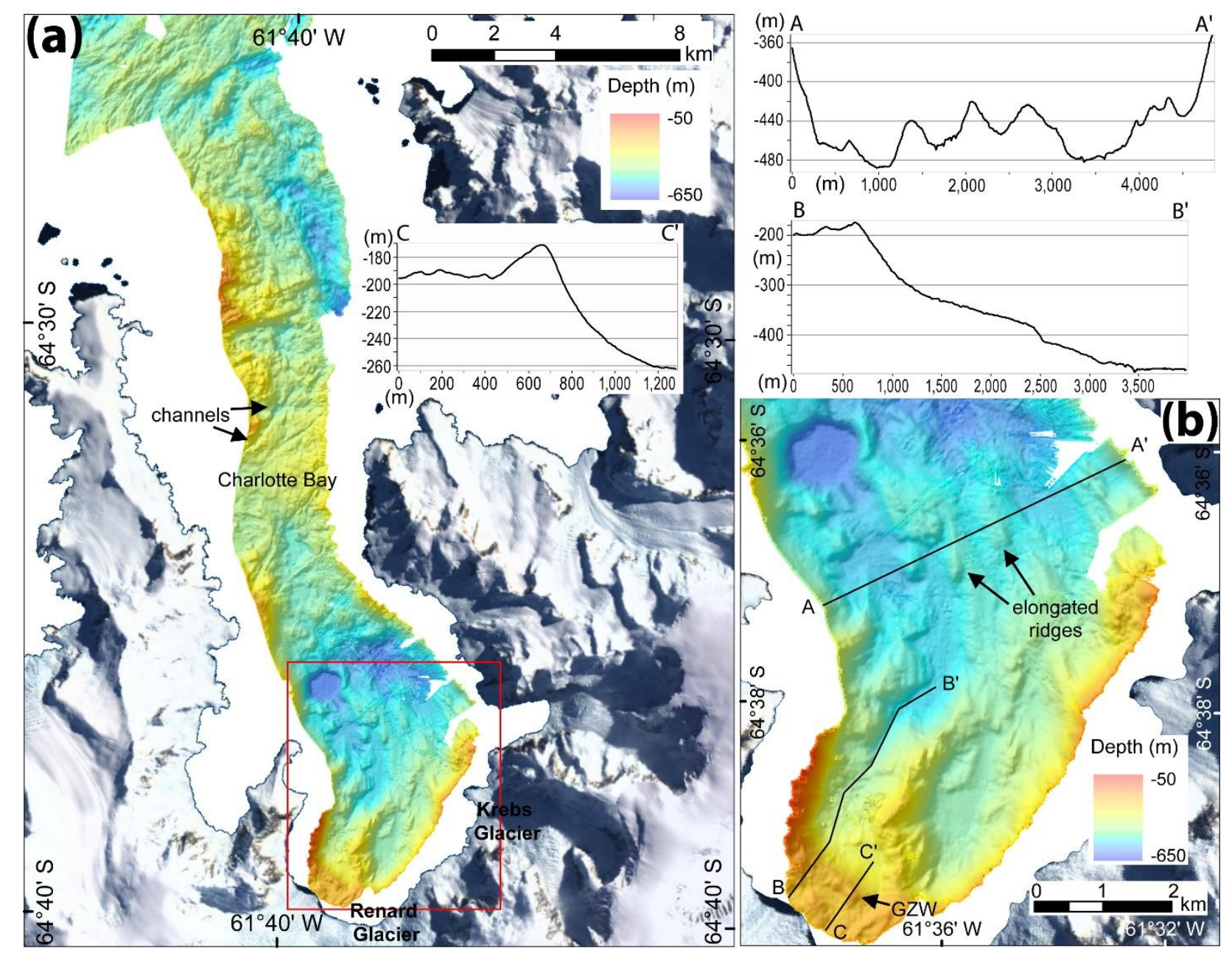

Figure 3: Charlotte Bay. 


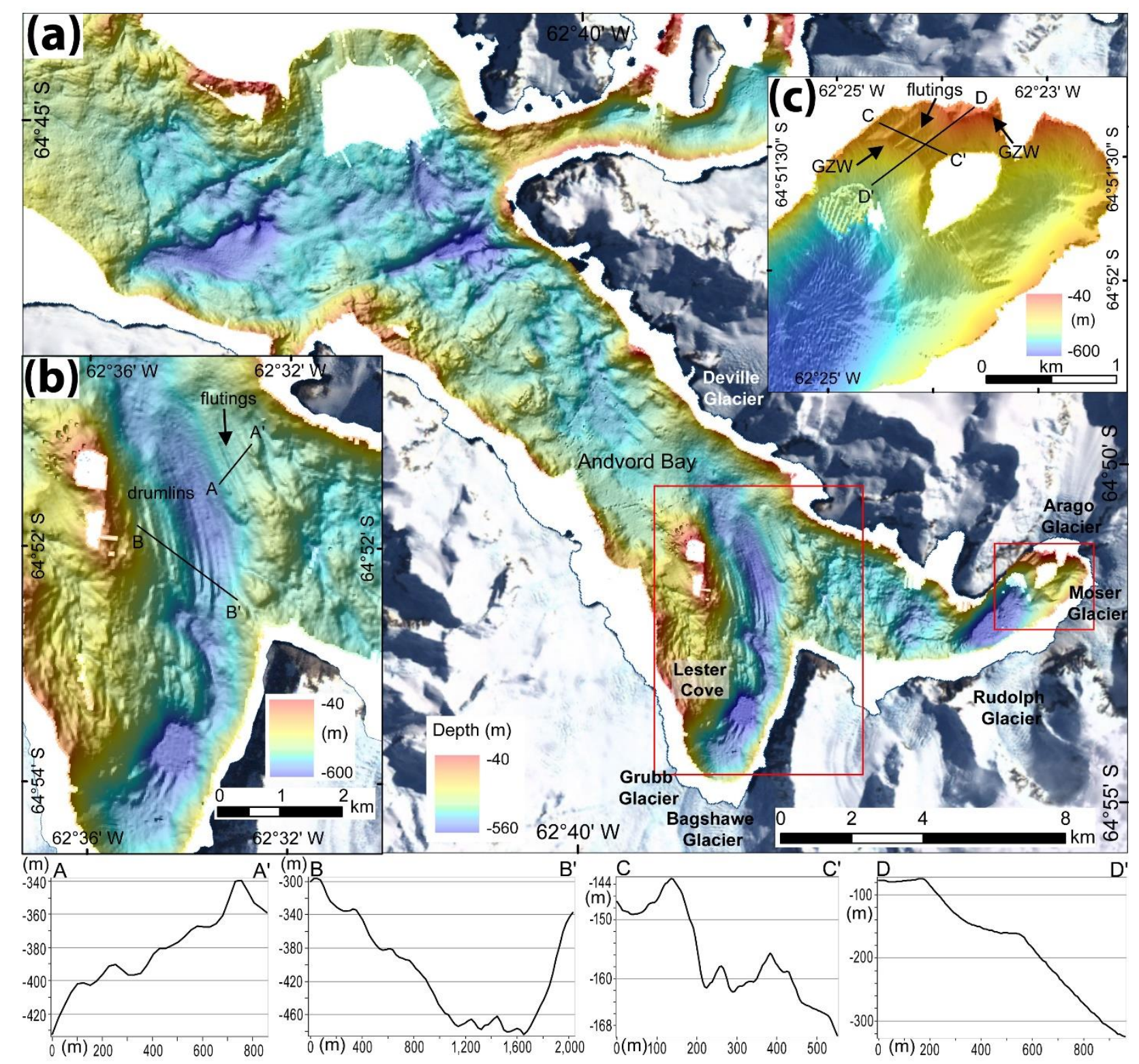

Figure 4: Andvord Bay, also showing Lester Cove (b) and Moser Glacier Cove (c). 


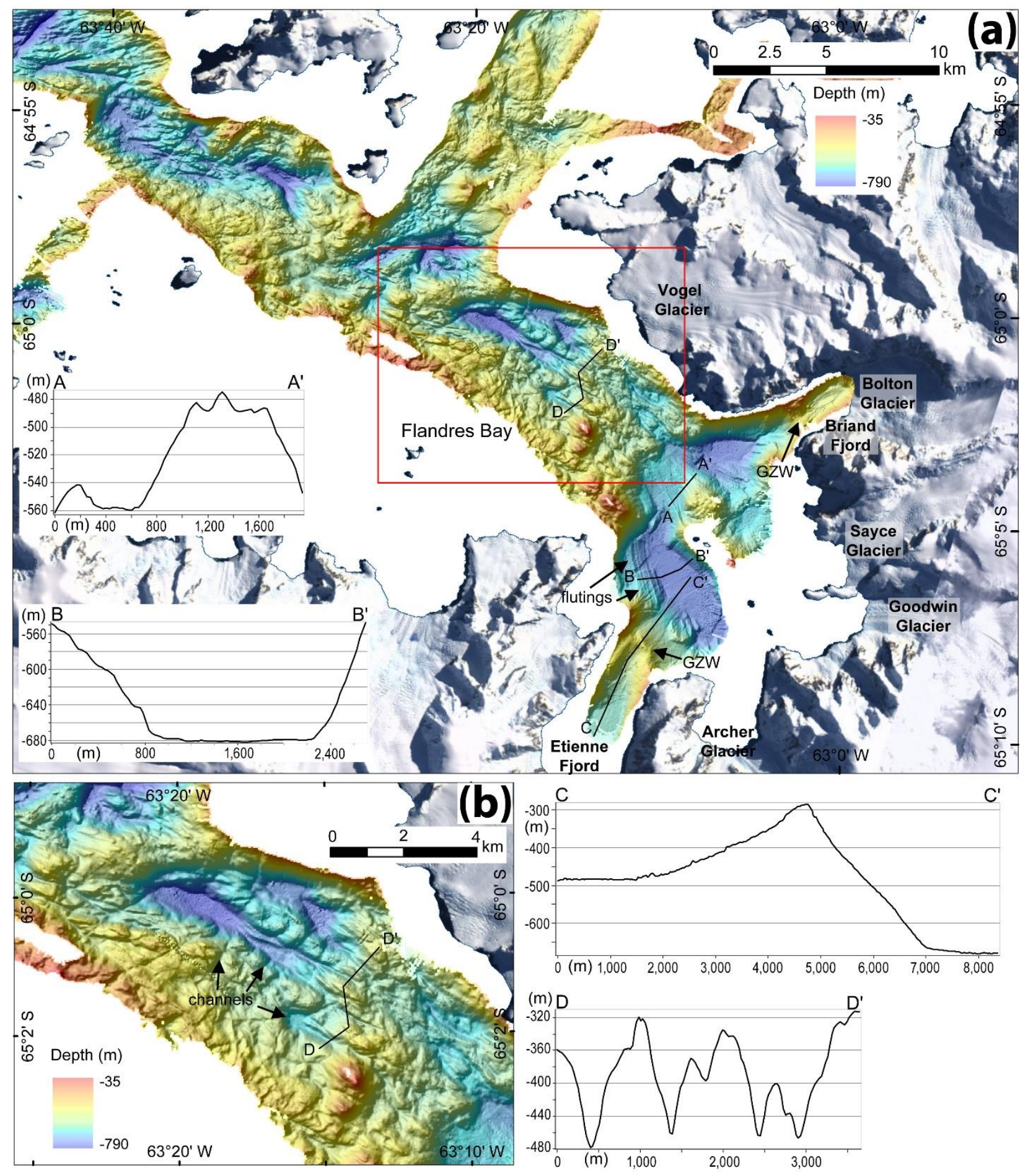

Figure 5: Flandres Bay, also showing Briand Fjord and Etienne Fjord. 


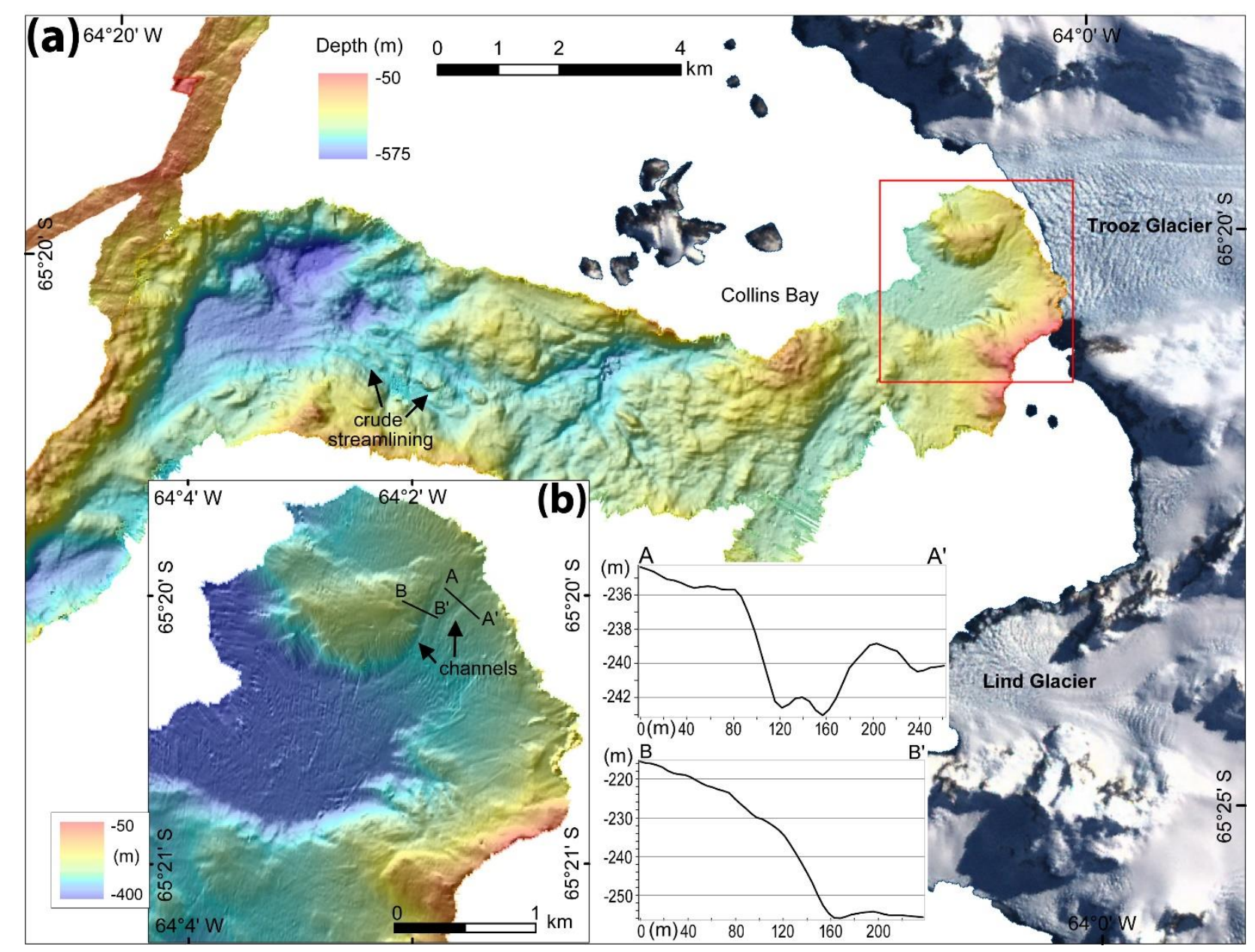

Figure 6: Collins Bay. 
Table 1. Measurements of western Antarctic Peninsula bays

\begin{tabular}{|c|c|c|c|c|c|c|c|c|c|}
\hline Bay Name & Longitude & Latitude & $\begin{array}{c}\text { Bay } \\
\text { Length } \\
(\mathbf{k m}) \\
\end{array}$ & $\begin{array}{c}\text { Bay } \\
\text { Width } \\
(\mathbf{k m}) \\
\end{array}$ & $\begin{array}{c}\text { Ratio } \\
\text { length/width }\end{array}$ & Bay Geometry & $\begin{array}{c}\text { Number of } \\
\text { Glaciers in Total }\end{array}$ & $\begin{array}{c}\text { Total Glacier } \\
\text { Catchment Area } \\
\left(\mathrm{km}^{2}\right) \\
\end{array}$ & $\begin{array}{c}\text { Bay Area } \\
\left(\mathbf{k m}^{2}\right)\end{array}$ \\
\hline Maxwell-Marian Cove & -58.769 & -62.213 & 3.8 & 1.2 & 3.2 & narrow & 1 & 14.99 & 4.868 \\
\hline Maxwell-Potter Cove & -58.687 & -62.232 & 4.3 & 1.6 & 2.7 & narrow & 1 & 20.26 & 7.093 \\
\hline Hope Bay & -57.046 & -63.407 & 5.8 & 2 & 2.9 & narrow & 4 & 27.36 & 11.448 \\
\hline Lapeyrere's unnamed & -63.286 & -64.37 & 4.6 & 1.8 & 2.6 & narrow & 2 & 60.05 & 7.959 \\
\hline Lapeyrere Bay & -63.284 & -64.421 & 11 & 2.6 & 4.2 & narrow & 7 & 271.06 & 32.496 \\
\hline Fournier Bay & -63.178 & -64.546 & 16 & 5.3 & 3.0 & narrow & 12 & 169.27 & 89.906 \\
\hline Advord Bay & -62.566 & -64.857 & 15 & 6 & 2.5 & narrow & 8 & 78.83 & 79.785 \\
\hline Andvord-Moser Gl. cove & -62.425 & -64.872 & 9 & 3.5 & 2.6 & narrow & 6 & 161.14 & 26.027 \\
\hline Flandres-Briand Fjord & -63.021 & -65.035 & 4.2 & 2 & 2.1 & narrow & 4 & 81.26 & 8.931 \\
\hline Flandres-Etienne Fjord & -63.234 & -65.171 & 8 & 3.2 & 2.5 & narrow & 7 & 244.5 & 24.587 \\
\hline Beascochea-Lever Gl. cove & -63.723 & -65.512 & 5.3 & 2.5 & 2.1 & narrow & 4 & 190.72 & 16.000 \\
\hline Beascochea Bay & -63.87 & -65.515 & 24 & 10 & 2.4 & narrow & 17 & 155.02 & 235.377 \\
\hline Beascochea-Funk Gl. cove & -63.756 & -65.58 & 4 & 2 & 2.0 & narrow & 2 & 157.77 & 7.874 \\
\hline Flandres Bay & -63.148 & -65.072 & 30 & 16 & 1.9 & broad & 23 & 774.64 & 453.073 \\
\hline Hughes-Brialmont Cove & -60.987 & -64.281 & 11 & 7.5 & 1.5 & broad & 5 & 842.53 & 85.500 \\
\hline Beascochea-Cadman Gl cove & -63.812 & -65.612 & 3 & 3 & 1.0 & broad & 1 & 307.04 & 8.915 \\
\hline \begin{tabular}{|l|} 
Andvord-Lester Gl. cove \\
\end{tabular} & -62.58 & -64.901 & 5 & 3.8 & 1.3 & broad & 2 & 276.33 & 16.997 \\
\hline Maxwell Bay & -58.848 & -62.229 & 15 & 10.4 & 1.4 & broad & 4 & 92.14 & 140.666 \\
\hline Collins Bay & -64.054 & -65.346 & 5.6 & 9.5 & 0.6 & open & 4 & 614.11 & 52.430 \\
\hline
\end{tabular}

5 Table 2. Seafloor features mapped in western Antarctic Peninsula bays

\begin{tabular}{|c|c|c|c|c|c|c|c|c|c|}
\hline Bay Name & Drumlins & GZW & Moraines & Basins & $\begin{array}{c}\text { Streamlined } \\
\text { Features }\end{array}$ & Flutings & Slope Failures & $\begin{array}{c}\text { Meltwater } \\
\text { Channels }\end{array}$ & $\begin{array}{c}\text { Total Features } \\
\text { per Bay }\end{array}$ \\
\hline Maxwell-Marian Cove & 0 & 1 & 4 & 3 & 0 & 5 & 10 & 7 & 30 \\
\hline Maxwell-Potter Cove & 0 & 1 & 11 & 1 & 0 & 0 & 0 & 0 & 13 \\
\hline Hope Bay & 0 & 2 & 10 & 2 & 0 & 0 & 0 & 5 & 19 \\
\hline Lapeyrere's unnamed & 0 & 1 & 0 & 1 & 0 & 0 & 10 & 5 & 17 \\
\hline Lapeyrere Bay & 0 & 1 & 0 & 2 & 3 & 7 & 40 & 23 & 76 \\
\hline Fournier Bay & 0 & 1 & 0 & 1 & 5 & 20 & 0 & 27 & 54 \\
\hline Charlotte Bay & 0 & 2 & 0 & 2 & 15 & 0 & 0 & 16 & 35 \\
\hline Andvord-Moser Gl. cove & 0 & 2 & 0 & 2 & 0 & 4 & 0 & 5 & 13 \\
\hline Flandres-Briand Fjord & 0 & 2 & 0 & 1 & 0 & 3 & 0 & 9 & 15 \\
\hline Flandres-Etienne Fjord & 0 & 1 & 0 & 1 & 0 & 0 & 0 & 4 & 6 \\
\hline Beascochea-Lever Gl. cove & 0 & 2 & 0 & 1 & 0 & 25 & 0 & 0 & 28 \\
\hline Beascochea Bay & 6 & 2 & 0 & 4 & 23 & 13 & 30 & 90 & 168 \\
\hline Beascochea-Funk Gl. cove & 0 & 1 & 0 & 1 & 0 & 17 & 0 & 33 & 52 \\
\hline Flandres Bay & 0 & 2 & 0 & 5 & 12 & 17 & 20 & 83 & 139 \\
\hline Hughes-Cierva Cove & 0 & 2 & 0 & 3 & 0 & 13 & 0 & 6 & 24 \\
\hline Beascochea-Cadman Gl. cove & 0 & 1 & 0 & 1 & 0 & 0 & 20 & 8 & 30 \\
\hline Andvord-Lester Gl. cove & 0 & 0 & 0 & 2 & 3 & 5 & 0 & 19 & 29 \\
\hline Maxwell Bay & 0 & 0 & 0 & 1 & 7 & 0 & 30 & 7 & 45 \\
\hline Collins Bay & 0 & 0 & 0 & 2 & 5 & 0 & 0 & 21 & 28 \\
\hline Total Features per Type: & 14 & 25 & 25 & 43 & 73 & 150 & 160 & 417 & 907 \\
\hline
\end{tabular}

